\title{
SPALLATION REACTIONS WITH NEUTRON-RICH AND NEUTRON-POOR NUCLEI
}

\author{
TH. AOUST \\ SCK-CEN, Boeretang 200, B-2400 Mol, Belgium \\ and \\ University of Liège, Physics Department \\ allée du 6 Aô̂t 17, bât. B5, B-4000 Liège 1, Belgium \\ E-mail: taoust@sckcen.be \\ J. CUGNON \\ University of Liège, Physics Department \\ allée du 6 Août 17, bât. B5, B-4000 Liège 1, Belgium \\ E-mail: cugnon@plasma.theo.phys.ulg.ac.be
}

\begin{abstract}
A renewed interest in spallation reactions in the $\mathrm{GeV}$ range has arisen, due to the recent advent of ADS projects for transmutation of nuclear waste. Most of investigations have dealt with proton-induced reactions on stable nuclei. Here, an exploratory theoretical investigation of spallation reactions on unstable nuclei is presented. The main issue is the dependence of the measurable quantities with the isospin of the target.
\end{abstract}

\section{Introduction}

Spallation reactions are very important for the development of radioactive beams ${ }^{1}$, in astrophysics ${ }^{2}$ and in cosmic-ray physics ${ }^{3}$. In recent years, there has been a renewed interest in proton-induced spallation reactions in the $\mathrm{GeV}$ incident energy range, due to the advent of projects of acceleratordriven systems (ADS) for transmutation of nuclear wastes (see e.g. Ref. ${ }^{4}$ ), leading to more systematic and more precise measurements. In parallel, a real improvement of the theoretical tools has taken place, especially in the frame of the EU HINDAS project ${ }^{5}$. These efforts have contributed to a more complete understanding of the spallation processes, strengthening the intranuclear-cascade (INC) plus evaporation approach. The process can be divided into two stages. In the first one the incident particle expells a few energetic particles by successive hadron-hadron collisions. In the second one 
the remaining, randomized, excitation energy is released by an evaporationlike process. In particular, it appeared that one of the best theoretical tools is provided by the Liège intranuclear cascade model INCL4 coupled to the K.-H. Schmidt ABLA evaporation-fission model. This approach, which is basically parameter-free, owing to a "self-consistent" determination of the stopping time, was shown recently to yield particularly good results for a large set of data for incident energies ranging from $200 \mathrm{MeV}$ to a few $\mathrm{GeV}^{6}$. These data include total reaction cross-sections, inclusive light particle production cross-sections, multiplicity distributions, residue production cross-sections and recoil distributions. Later on, this approach was shown to be largely successful in the description of spallation reactions at lower incident energy, down to a few tens of $\mathrm{MeV}$, after small changes in the treatment of the Pauli principle ${ }^{7}$.

The HINDAS project has considerably improved the measurements of the isotopic cross-sections. Usually, the latter are performed in protoninduced reactions by activity (basically $\gamma$-ray) measurements. In the HINDAS project, reverse kinematics experiments have been performed: stable nuclei bombard an hydrogen target and forward-flying residues are analyzed by a magnetic isotopic spectrometer. Individual isotope production cross-section can be determined, in constrast to the activity method which can only access to cumulated cross-sections.

This method could in principle be extended to accelerated non-stable nuclei, provided the intensity of the beam is sufficiently large. Here, we present an exploratory theoretical investigation of spallation reactions on neutron-rich and neutron-poor nuclei. We will use the same approach as in Ref. ${ }^{6}$, except for an improved treatment of the nuclear mean field. The main issue is to know whether there are qualitative differences with spallation reactions on ordinary nuclei. In particular, the following questions will be studied: (i) What is the evolution of the neutron multiplicities with the target neutron (or proton) excess? (ii) Is the shape of the residue mass spectra qualitatively modified?

\section{The theoretical approach}

Our INC+evaporation model combines the INCL4 version of the Liège intranuclear-cascade model ${ }^{6}$ and the ABLA evaporation-fission code of K.-H. Schmidt ${ }^{8,9}$. It is described in Ref. ${ }^{6}$. We briefly recall the main features. The collision mechanism is assumed to proceed from a succession of binary collisions (and decays) well separated in space and time. 
The fate of all particles is followed as time evolves. Particles travel along straight-line trajectories until two of them reach their minimum distance of approach, in which case they can be scattered provided the value of this distance is small enough, or until they hit the border of the potential well, supposed to describe the nuclear target mean field. Additional features are: (1) initial positions of target nucleons are taken at random in the spherical nuclear target volume with a smooth surface; (2) an improved treatment of the Pauli blocking is introduced; (3) inelasticity is introduced through reactions involving pion's and delta's (4) isospin degrees of freedom are introduced for all types of particles; (5) the cascade code is stopped at a time determined by the code itself, when the emission pattern becomes evaporative; (6) the ABLA code has a sophisticated evaporation-fission competition with viscosity effects.

In the standard INCL4 version, although neutron and protons are distinguished, they experience the same nuclear average potential, except, of course, for the Coulomb external potential. Here, we introduced isospin and energy-dependent potentials, following the optical-model phenomenology (details can be found in Ref. ${ }^{10}$ ).

\section{Observable quantities}

\subsection{Introduction}

We present calculations for particle and residue cross-sections. Altough spallations reactions with unstable nuclei will probably be realized in reverse kinematics, we present results as in direct kinematics, for the sake of comparison. We will consider two types of targets: $P b$ and $S n$ isotopes.

\subsection{Particle production cross-section}

Average proton and neutron multiplicities are given in Table 1 for $p$-induced reactions on $S n$ and $P b$ reactions at $1 \mathrm{GeV}$. In each case, a neutron-poor and a neutron-rich targets are compared with a stable isotope.

The most remarkable, but rather expected, result is the enhancement (reduction) of the neutron multiplicity for the neutron-rich (-poor) isotopes. Less obviously, this enhancement (reduction) is less marked, even proportionally, for the emission during the cascade stage (mainly $E_{n} \leq 20$ $\mathrm{MeV})$. The large part of the effect is coming from the evaporation stage. The cascade emission leaves a remnant which is not far from the target in the $(\mathrm{N}, \mathrm{Z})$ plane: as a result a neutron-rich remnant evaporates first 
a few neutrons before reaching the so-called "evaporation corridor" on the proton-rich side of the valley of stability, parallel to the stability line, where a balance between proton emission (favoured by smaller separation energies) and neutron emission (favoured by the absence of Coulomb barrier) settles.

\begin{tabular}{|c|c|c|c|}
\hline \multicolumn{4}{|c|}{$p+S n$} \\
\hline & ${ }^{100} \mathrm{Sn}$ & ${ }^{124} S n$ & ${ }^{134} S n$ \\
\hline$<n>, E_{n}<2 \mathrm{MeV}$ & 0.34 & 1.87 & $\overline{2.92}$ \\
\hline$<n>, 2 \mathrm{MeV}<E_{n}<20 \mathrm{MeV}$ & 1.41 & 5.19 & 8.01 \\
\hline$<n>, E_{n}>20 M e V$ & 1.71 & 2.25 & 2.64 \\
\hline$<n>$ & 3.42 & 9.30 & 13.57 \\
\hline$<p>$ & 7.40 & 3.30 & 2.50 \\
\hline$S_{n}(\mathrm{MeV})$ & 17.65 & 9.10 & $\overline{3.90}$ \\
\hline$S_{p}(\mathrm{MeV})$ & 2.80 & 10.70 & 16.2 \\
\hline \multicolumn{4}{|c|}{$\overline{p p+P b}$} \\
\hline & ${ }^{182} \mathrm{~Pb}$ & ${ }^{208} \mathrm{~Pb}$ & ${ }^{212} \mathrm{~Pb}$ \\
\hline$<n>, E_{n}<2 M e V$ & 1.39 & 3.50 & $\overline{3.80}$ \\
\hline$<n>, 2 \mathrm{MeV}<E_{n}<20 \mathrm{MeV}$ & 3.44 & 7.87 & 8.50 \\
\hline$<n>, E_{n}>20 \mathrm{MeV}$ & 2.20 & 2.61 & 2.73 \\
\hline$<n>$ & 7.10 & 13.98 & 15.03 \\
\hline$<p>$ & 5.90 & 3.10 & 2.90 \\
\hline$S_{n}(\mathrm{MeV})$ & 11.75 & 7.40 & 5.10 \\
\hline$S_{p}(\mathrm{MeV})$ & 1.30 & 8.00 & 8.80 \\
\hline
\end{tabular}

There is, of course, a symmetric effect on the proton multiplicities. It is less dramatic in absolute values, but more dramatic in proportion.

\subsection{Residue production cross-section}

The residue mass spectra for $p$-induced reactions on $S n$ targets are given in Fig. 1. The abscissa $x$ is equal to 120 minus the mass loss. It is appropriate to compare the so-called fragmentation peaks, corresponding to the residues which are created by evaporation $(x \gtrsim 70)$. As expected, the fragmentation peak is broader for ${ }^{134} \mathrm{Sn}$, mainly because starting from the neutron-rich side of the valley of stability, the system can evaporate more nucleons before reaching the evaporation corridor. The fission contribution is very small in this case. It corresponds to the $x \lesssim 70$ part of the spectrum. It is only significant for ${ }^{100} \mathrm{Sn}$, which has the largest fissility parameter. 


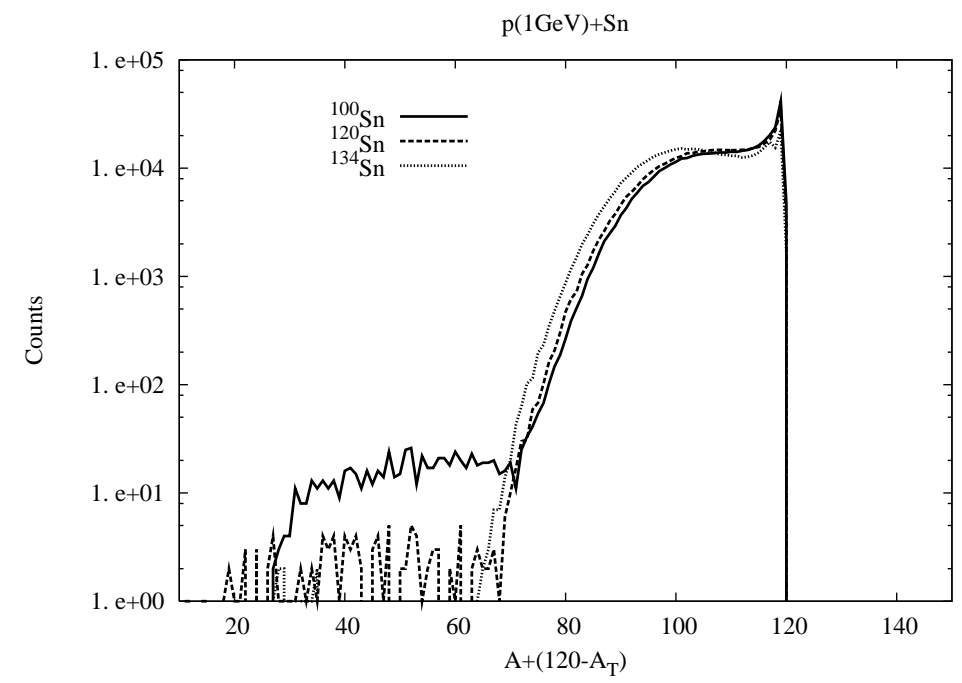

Figure 1. Residue mass spectra for proton-induced reactions on three $S n$ targets at 1 $\mathrm{GeV}$. Note that the horizontal scale has been chosen such that the isobars corresponding to the same mass loss appear at the same place for the three systems.

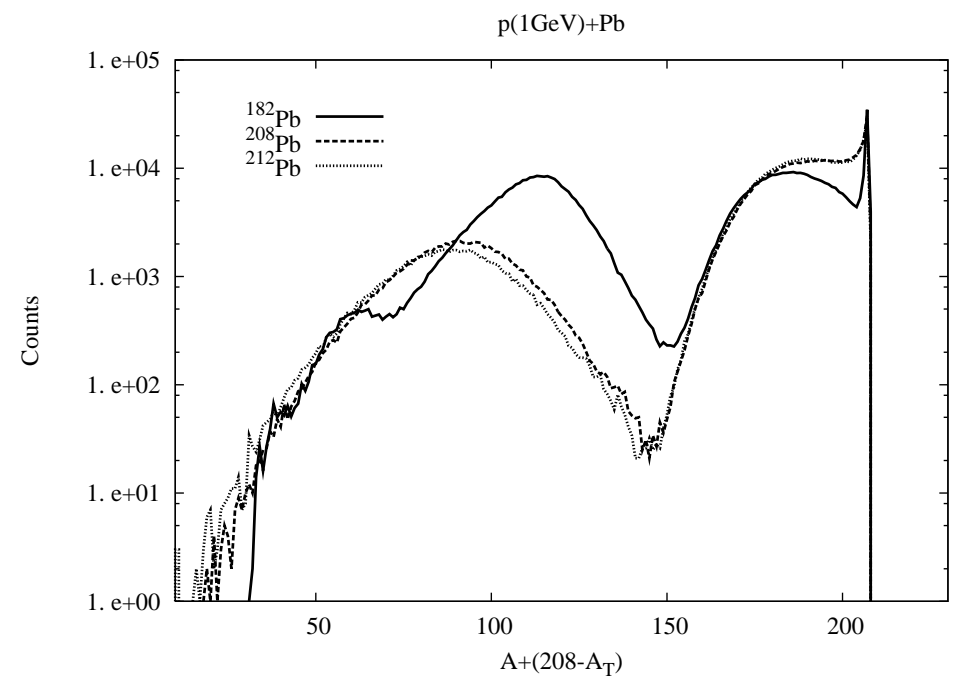

Figure 2. Same as Fig. 1 for three $P b$ targets.

The residue mass spectra for three isotopes of $P b$ are given in Fig. 2. In this case, the fragmentation peaks have roughly the same width. In fact, 
they are practically identical for ${ }^{208} \mathrm{~Pb}$ and ${ }^{212} \mathrm{~Pb}$. The shape is different for ${ }^{182} \mathrm{~Pb}$, but this is mainly linked with the most striking result of Fig. 2, namely the large fission cross-section for this target. This, of course, is due to the large fissility parameter of the remnants and leads to a large depopulation of the mass spectrum around mass loss $\approx 5-6$. As for the other targets, a peak exists for mass loss of one mass unit. This peak arises because peripheral collisions lead to small excitation energy; for smaller impact parameters, more excitation energy is left in the target remnant and leads, either to important evaporation or, as in this particular case of ${ }^{182} \mathrm{~Pb}$, to fission.

\section{Conclusion}

We have presented here exploratory calculations for spallation reactions on neutron-rich and neutron-poor nuclei.Neutron and proton multiplicities can be substantially changed. The shape of the residue mass spectra is not drastically different from the ordinary target case, except for fission.

\section{References}

1. I. Tanihata, On the possible use of secondary radioactive beams, in Treatise on Heavy-Ion Science, vol. 8, ed. by D.A. Bromley, Plenum Press, (1989).

2. T. K. Gaiser, Cosmic Rays and Particle Physics, Cambridge University Press, (1992).

3. M. Longair, High Energy Astrophysics, vol. 1 and 2, Cambridge University Press, (1997).

4. W. Gudowski, Nucl. Phys. A654, 436c (1999).

5. A. Koning et al, HINDAS, A European Nuclear Data Program for AcceleratrDriven Systems, Tsukuba Conference, 2002; J.-P. Meulders et al, HINDAS final report, to be published in EU publications 2004.

6. A. Boudard, J. Cugnon, S. Leray and C. Volant, Phys. Rev. C66, 044615 (2002).

7. J. Cugnon and P. Henrotte, Eur. Phys. J. A 16, 393 (2003).

8. J.-J. Gaimard and K.-H. Schmidt, Nucl. Phys., A531 709 (1991).

9. A. R. Junghans, M. de Jong, H. G. Clerc, A. V. Ignatyuk, G. A. Kudyaev and K.-H. Schmidt, Nucl. Phys. A629, 635 (1998).

10. Th. Aoust and J. Cugnon, to be published in Eur. Phys. J. A (2004). 